\section{Bildung für Nachhaltige Entwicklung in Reallaboren}

\author{
Die bildungsbezogenen Angebote des \\ „Energielabors Tübingen“ in der Kinder- \\ Uni Tübingen
}

\author{
von Marius Albiez, Andri König und Thomas \\ Potthast, Universität Tübingen
}

\begin{abstract}
In Reallaboren forschen und lernen Wissenschaftlerlnnen und Bürgerlnnen gemeinsam für Nachhaltige Entwicklung. Welche Rolle Bildung für Nachhaltige Entwicklung (BNE) dabei spielen kann, wird im vorliegenden Beitrag am Beispiel der Angebote des „Energielabor Tübingen" für die Kinder-Uni diskutiert. BNE trägt im Energielabor bei allen Beteiligten dazu bei, Bewusstsein und Kompetenzen für Nachhaltige Entwicklung weiter zu entwickeln. Zu den Beteiligten zählen auch Kinder, die als „nächste Generation“ und Multiplikatoren für Nachhaltige Entwicklung Adressaten der BNE sind. Sie sollten stärker als bislang in Reallabore eingebunden werden.
\end{abstract}

In „Urban Transition Labs“, scientists and citizens research and learn together to promote sustainable development. This paper discusses what role Education for Sustainable Development (ESD) could play in Urban Transition Labs. It uses the contributions of the project „Energy Lab Tübingen" to the Children's University Tübingen as an example. Here, ESD creates and develops awareness and skills in favour of sustainable development. This concerns all participants, including children. Children are the "next generation” and multipliers for sustainable development. As such, they are addressees of ESD, and they should be integrated deeper into project forms like Urban Transition Labs.

\section{1 Überblick}

Im Folgenden wird das „Energielabor Tübingen"1 vorgestellt und die Rolle von BNE in diesem Reallabor diskutiert. Erfahrungen aus zwei Veranstaltungen im Rahmen der Kinder-Uni Tübingen werden erläutert sowie deren Bedeutung für das Projekt „Energielabor“ erörtert. Die dabei gewonnenen Erkenntnisse werden abschlie- ßend in den übergreifenden Diskurs zu Reallaboren eingeordnet.

\section{Das Energielabor Tübingen}

Das „Energielabor Tübingen“ zielt darauf ab, gemeinsam mit BürgerInnen in einem partizipativen Prozess die Energiewende in Tübingen voranzutreiben, indem Potenziale sowie Perspektiven für eine nachhaltige Energieversorgung und -nutzung insbesondere mit Bezug auf regenerative Energiequellen erforscht und praktisch erprobt werden. Das inter- und transdisziplinäre Projekt wird gemeinsam vom Geographischen Institut (Universität Tübingen, federführend), dem Internationalen Zentrum für Ethik in den Wissenschaften (IZEW; Universität Tübingen), dem Institut für Energiewirtschaft und Rationelle Energieanwendung (Universität Stuttgart) sowie von außeruniversitären Partnern durchgeführt, $\mathrm{zu}$ denen die Universitätsstadt Tübingen, die imakomm Akademie, die Stadtwerke Tübingen, das Umweltzentrum Tübingen e.V. sowie der Bund für Umwelt und Naturschutz, Regionalverband Neckar-Alb zählen.

Die „Energiewende“ wird von Teilen der Öffentlichkeit, der Politik und der Wissenschaft oftmals auf die technische Transformation (Effizienz und Konsistenz) des Energiesystems verkürzt, um aus der Atomenergie auszusteigen und die Umstellung von fossilen Energiequellen auf erneuerbare rasch voranzutreiben. Ebenso wichtig für das Gelingen der Energiewende sind jedoch soziale Aspekte, die unter anderem Bildungsprozesse, das individuelle NutzerInnenverhalten sowie Partizipation und den Umgang mit Konflikten umfassen. Neben Effizienz und Konsistenz ist die Frage nach dem richtigen Maß, also der Suffizienz als dritter, gleichberechtigter Leitlinie einer Nachhaltigen Entwicklung (NE) zu beachten. Insgesamt gilt es Gerechtigkeitsfragen zu erforschen, da diese die Grundlage des normativen Leitbilds NE sind. Vor diesem Hintergrund werden im Energielabor soziale, technische und ethische Fragestellungen zusammengedacht und unter dem Begriff „nachhaltige Energiewende" bearbeitet. Zu klären, was diese im Einzelnen bedeutet, wie sie vor Ort gestaltet werden kann und wie die Erkenntnisse auf an- 
dere Städte und Kommunen übertragen werden können, sind Kernanliegen des Reallabors.

Dazu werden die soziotechnischen Potenziale einzelner regenerativer Energieträger in ausgewählten Tübinger Stadtquartieren erfasst und kartographisch aufgearbeitet. Des Weiteren werden Strategiemodelle zur Verbesserung der Energieeffizienz auf Quartiersebene erarbeitet, etwa mithilfe von Energieflüssen und -bilanzen. Zudem werden bereits vorhandene Initiativen und Akteure der Energiewende in Tübingen mit WissenschaftlerInnen und der Bürgerschaft vernetzt, um gemeinsam „Wendepunkte“ zu erarbeiten und umzusetzen. Als Wendepunkte werden im Projekt diejenigen Realexperimente (Schneidewind 2014) bzw. deren Elemente und soziotechnischen Innovationen bezeichnet, die zu einer nachhaltigen Energiewende in Tübingen beitragen. Gemäß dem transformativen Wissenschaftsverständnis (WBGU 2011, S. 23) greifen die WissenschaftlerInnen des Energielabors in die Wendepunkt-Experimente ein und arbeiten so System-, Ziel- und Handlungswissen einer nachhaltigen Energiewende heraus.

Transformationsprozesse erfordern aber transformatives Bewusstsein und entsprechende Fähigkeiten der Akteure, die mittels Bildung für Nachhaltige Entwicklung vermittelt und eingeübt werden können und sollen.

\section{Bildung für Nachhaltige Entwicklung für Studierende, Forschende und Kinder im Energielabor Tübingen}

Bildung für Nachhaltige Entwicklung (BNE) ist ein wesentlicher Bestandteil im Energielabor Tübingen, um die sozialen, technischen und ethischen Fragestellungen angemessen angehen zu können. BNE dient der Bewusstseinsbildung und Kompetenzentwicklung der beteiligten Akteure, „welche wiederum Voraussetzung[en] für eine nachhaltige Entwicklung" (Alisch et al. 2015, S. 31; de Haan 2006), hier mit Bezug auf die Energiewende, sind.

Im Energielabor Tübingen geht es auch darum, ,über soziale Dynamiken und Prozesse zu lernen" (Schneidewind 2014, 3). Hier findet also BNE statt. Zielgruppen sind die involvierten WissenschaftlerInnen mit unterschiedlicher dis- ziplinärer Ausrichtung, beteiligte PraxispartnerInnen, Studierende und die Öffentlichkeit.

Studierende sollen eine Schlüsselrolle innerhalb des Reallabors einnehmen, da sie Teil des Wissenschaftsbetriebs sind und zugleich als BürgerInnen sowie als Praxisakteure innerhalb von Initiativen auftreten können. Mitgestaltung ebenso wie Reflexion geschieht unter anderem im Rahmen von Lehrveranstaltungen oder bei der Bearbeitung von Abschlussarbeiten.

Die BNE-Aktivitäten des Energielabors liefern Einsichten und Kompetenzen auch für die im Reallabor tätigen WissenschaftlerInnen, etwa wenn es um die gemeinsame Entwicklung von Forschungsthemen oder bedarfsgerechten Nachhaltigkeitsindikatoren geht.

BNE sollte sich allerdings an alle Altersgruppen richten, also auch an Kinder. Sie haben Bedürfnisse und ein Recht ihre Zukunft mitgestalten zu können, werden jedoch im bisherigen Diskurs zu BNE in Reallaboren kaum berücksichtigt.

\section{Bildungsangebote des Energielabors Tübingen in der Kinder-Uni}

Die BNE-Aktivitäten des Energielabors Tübingen im Rahmen der Kinder-Uni wurden vom IZEW inhaltlich konzipiert und durchgeführt. Das verfolgte Ziel war, Kinder an das Thema Energiewende heranzuführen.

Die Universität Tübingen besitzt langjährige Erfahrung mit dem dort erstmals erprobten Format „Kinder-Uni“ als Ringvorlesung mit wechselnden Vorträgen und Themen (Janßen/ Steuernagel 2003). Im Sommersemester 2016 brachte Thomas Potthast, unterstützt von Andri König, in einer Vorlesung BNE und transformative Elemente zum Thema Nachhaltige Entwicklung ein. Der Titel lautete „Warum haben wir die Erde nur von unseren Kindern geliehen?" Durch stärker dialogische Elemente im Vortrag konnten jeweils ca. 15 der etwa 150 zuhörenden Kinder aktiv mit einbezogen werden. Eine Maßnahme hierfür war, dass der Vortragende die ,räumliche Schranke" zwischen ihm und dem Auditorium durchbrach, indem er sich bei dem Gespräch mit den Kindern im gesamten Hörsaal bewegte und sich Antworten der Kinder auf seine Fragen direkt an deren Platz abholte. Weitere Anregungen 
zur Aktivierung wurden von Krüger (2012, S. 132) übernommen, so die Idee, dass die Kinder sich einen Brief schreiben, in dem sie ihre künftigen Aktivitäten zur Nachhaltigen Entwicklung konkret beschreiben.

Ein anderes Format wurde im Rahmen des Kinder-Uni-Forschertags eingesetzt. Kinder und WissenschaftlerInnen trafen sich zur Veranstaltung „Woher kommt unser Strom und wofür brauchen wir ihn?", um sich gemeinsam mit Themen einer nachhaltigen Energiewende zu beschäftigen. Die Veranstaltung wurde von Marius Albiez und Andri König konzipiert und durchgeführt. An der rund zweistündigen Veranstaltung nahmen insgesamt fünf Kinder zwischen sieben und elf Jahren teil. Der Ablauf und die Beobachtungen der jeweiligen Veranstaltungsschritte können in Tabelle 1 nachvollzogen werden.

BNE bedeutet in diesem Zusammenhang auch mit Kindern zu philosophieren (Krüger 2012): Themen der Nachhaltigen Entwicklung haben stets eine ethische Dimension. So wird z. B. mit Kindern diskutiert, wie wir zukünftig mit Energie umgehen sollen und warum wir unsere Konsumweise ändern müssen. Dabei kommt es - meist unbemerkt - zu sog. „gemischten Urteilen", welche unter Einbezug empirischer Fakten und normativer Prämissen entstehen (Potthast 2015). Die Sensibilisierung für und Diskussion von diesen oftmals impliziten normativen Prämissen im Nachhaltigkeitsdiskurs ist eine Aufgabe der BNE und Ethik, nicht zuletzt im Praxiskontext der Reallabore, in denen solche impliziten gemischten Urteile transparent gemacht werden können und sollen.

\section{Was bedeutet die Einbindung von Kindern für Reallabore?}

Selbstverständlich können die im Zuge der Veranstaltungen gewonnenen ersten Erfahrungen nicht einfach verallgemeinert werden. Gleichwohl sollen einige grundsätzliche Überlegungen zur Rolle von BNE und Kindern in Reallaboren zur Diskussion gestellt werden.

Da Nachhaltige Entwicklung alle Lebensbereiche betrifft, sollte auch BNE ein möglichst breites Spektrum an Personen in den Blick nehmen. Zugleich ist genauer zu bestimmen, an wen die jeweiligen Bildungsaktivitäten adressiert sind und welche Akteure eingebunden werden. Wie oben erwähnt, sind Forschende und andere Projektbeteiligte ebenso wie Studierende geeignete Adressaten für BNE-Maßnahmen in Reallaboren. Dies durchbricht vermeintlich festgelegte Rollen als ExpertInnen oder Lernende und kann zu komplexeren gegenseitigen LehrLernprozessen führen.

Aber auch Kinder können und sollten viel stärker in Reallaboraktivitäten eingebunden werden als dies bisher der Fall ist. Dies bietet die Gelegenheit, eine Kultur der Nachhaltigkeit (Parodi et al. 2016) möglichst früh zu diskutieren, auszutesten und zu erleben. Kinder können als Multiplikatoren auftreten, indem sie den Gedanken einer Nachhaltigen Entwicklung in Bereiche und Institutionen tragen, zu denen die im Reallabor Tätigen nur eingeschränkten Zugang haben. Zu nennen wären hier Kindertagesstätten, Schulen, Sportvereine, aber auch die Familie des jeweiligen Kindes sowie deren Bekanntenkreis.

Die beiden vorgestellten BNE-Aktivitäten im Rahmen der Kinder-Uni sind nur zwei BNEFormate von vielen denkbaren, tragen jedoch auch zu einer grundsätzlichen Annäherung zwischen Bürgerschaft und Wissenschaft bei; insofern sind es auch Partizipationsformate. Interessant ist dabei, dass das Format in Reallaboren prinzipiell die Möglichkeit bietet, auch disziplinäre Wissenschaft einzubinden. Zudem kann an weitere Lehrformate angeknüpft werden, die im bisherigen Reallabordiskurs noch eine untergeordnete Rolle spielen, wie beispielsweise Service Learning, also der Verknüpfung zwischen gesellschaftlichem Engagement und universitärer Lehre. So wäre auch eine Zusammenarbeit von Studierenden und Kindern innerhalb des Reallabors denkbar. Dies soll im weiteren Verlauf des Projekts „Energielabor Tübingen“" ausprobiert werden.

\section{Anmerkung}

1) Weitere Informationen finden sich hier: http://www. uni-tuebingen.de/de/79692 (download 14.12.16); http://www.gemeinsam-zur-energiewende.de (download 14.12.16) 
Tab. 1: Darstellung des Ablaufs und der Beobachtungen im Rahmen der Veranstaltung „Woher kommt unser Strom und wofür brauchen wir ihn?“", 2.7.2016

\begin{tabular}{|c|c|c|c|}
\hline $\begin{array}{l}\text { Elemente der } \\
\text { Lehrveranstaltung }\end{array}$ & Ablauf & Bildungsziel & Beobachtungen \\
\hline Vorstellungsrunde & $\begin{array}{l}\text { Gemeinsame Diskussion: } \\
\text { „Was habe ich heute Mor- } \\
\text { gen gefrühstückt? Wie bin } \\
\text { ich hergekommen, mit wel- } \\
\text { chem Verkehrsmittel?“ }\end{array}$ & $\begin{array}{l}\text { Bezüge herstellen zum } \\
\text { Alltag der Kinder, } \\
\text { Sensibilisierung über } \\
\text { die Bedeutung des } \\
\text { eigenen Verhaltens und } \\
\text { Energiekonsums. } \\
\text { Aufbau eines } \\
\text { Vertrauensverhältnisses } \\
\text { zwischen Lehrenden und } \\
\text { teilnehmenden Kindern } \\
\text { sowie zwischen den } \\
\text { Kindern untereinander. }\end{array}$ & $\begin{array}{l}\text { Abstraktionsvermögen } \\
\text { seitens der Kinder ist (im } \\
\text { Ansatz) vorhanden. } \\
\text { Kinder zogen Rückschlüsse } \\
\text { zum eigenen Alltag und } \\
\text { dessen Bedeutung für Ener- } \\
\text { gieverbräuche. }\end{array}$ \\
\hline $\begin{array}{l}\text { Vorstellung und Durchfüh- } \\
\text { rung eines Gedankenexperi- } \\
\text { ments (Krüger 2012, S. 70) }\end{array}$ & $\begin{array}{l}\text { Vorstellung und Beschrei- } \\
\text { bung, wie der eigene Alltag } \\
\text { im Mittelalter ausgesehen } \\
\text { haben könnte. } \\
\text { Anschließende Diskussion }\end{array}$ & $\begin{array}{l}\text { Erste Annäherung an Wis- } \\
\text { senschaft und Forschung. } \\
\text { Erste Annäherung an Sys- } \\
\text { temwissen im Bereich nach- } \\
\text { haltiger Energieversorgung }\end{array}$ & $\begin{array}{l}\text { Die Kinder identifizierten } \\
\text { verschiedene Felder, die mit } \\
\text { der Nutzung von Energie } \\
\text { zusammenhängen. } \\
\text { Die Nutzung von Strom für } \\
\text { technische Geräte wurde als } \\
\text { Normalfall empfunden. } \\
\text { (Wecker wurde im Gedan- } \\
\text { kenexperiment durch einen } \\
\text { Hahn ersetzt, nicht jedoch } \\
\text { durch eine mechanische } \\
\text { Lösung) }\end{array}$ \\
\hline $\begin{array}{l}\text { Brainstorming-Übungen, } \\
\text { Visualisierungen, Experi- } \\
\text { mente }\end{array}$ & $\begin{array}{l}\text { Auslegen einer gezeichne- } \\
\text { ten Steckdose, die von je- } \\
\text { weils zwei Pfeilen flankiert } \\
\text { wurde (hinein und hinaus). } \\
\text { Auf Karteikarten eine } \\
\text { Erzeugungsart sowie ein } \\
\text { Anwendungsfeld für Strom } \\
\text { notieren. } \\
\text { Ergebnisse wurden den } \\
\text { jeweiligen Pfeilen zugeord- } \\
\text { net. (Krüger 2012, S. 69) } \\
\text { Gemeinsames Basteln eines } \\
\text { Aufwindkraftwerkmodells. }\end{array}$ & $\begin{array}{l}\text { Kinder spielerisch an Sys- } \\
\text { temwissen zu elektrischer } \\
\text { Energie heranführen. }\end{array}$ & $\begin{array}{l}\text { Die Kinder verfügen über } \\
\text { Grundkenntnisse der } \\
\text { Energieerzeugung und } \\
\text { Erzeugungsarten wie ,Was- } \\
\text { serkraftwerk“, Solaranlage } \\
\text { auf dem Dach, Windkraft, } \\
\text { Verbrennung, ,Atomkraft- } \\
\text { werk“. } \\
\text { Die Stromgewinnung } \\
\text { mittels erneuerbarer Ener- } \\
\text { gieträger wurde wesentlich } \\
\text { häufiger genannt als fossile } \\
\text { oder nukleare. }\end{array}$ \\
\hline Abschlussdiskussion & $\begin{array}{l}\text { Abschlussdiskussion in der } \\
\text { Gruppe: Wann, wo und in } \\
\text { welchem Maß benötigen die } \\
\text { Kinder Strom in ihrem All- } \\
\text { tag? In welchen Fällen kann } \\
\text { darauf verzichtet werden? } \\
\text { Anschließend sollten ge- } \\
\text { meinsam Ideen und Maß- } \\
\text { nahmen zur Reduktion des } \\
\text { eigenen Energiebedarfs } \\
\text { entwickelt werden. }\end{array}$ & $\begin{array}{l}\text { Reflexion über das eigene } \\
\text { Maß } \\
\text { Maßnahmen entwickeln } \\
\text { zur Reduktion des eigenen } \\
\text { Energiebedarfs. }\end{array}$ & $\begin{array}{l}\text { Kinder konnten unterschied- } \\
\text { liche Möglichkeiten nennen, } \\
\text { den eigenen Stromver- } \\
\text { brauch zu senken. Sie hatten } \\
\text { jedoch Schwierigkeiten, das } \\
\text { eigene Maß zu definieren }\end{array}$ \\
\hline
\end{tabular}

Quelle: Eigene Darstellung 


\section{Literatur}

Alisch, J.M.; Bühr, M.; Holzbaur, U., 2015: Nachhaltigkeit im Kinder-,,Garten“. Raum- und erlebnisorientierte Konzepte in der frühkindlichen Bildung für nachhaltige Entwicklung. Aachen

de Haan, G., 2006: Bildung für nachhaltige Entwicklung - ein neues Lern- und Handlungsfeld. In: UNESCO heute 1 (2006), S. 4-8

Janßen, U.; Steuernagel, U., 2003: Die Kinder-Uni. Forscher erklären die Rätsel der Welt. Stuttgart

Krüger, B., 2012: Wie wollen wir leben? Kinder philosophieren über Nachhaltigkeit. München

Parodi, O.; Albiez, M.; Meyer-Soylu, S. et al., 2016 : Das „Quartier Zukunft - Labor Stadt“: ein reales Reallabor. In: Hahne, U.; Kegler, H. (Hg.): Resilienz. Stadt und Region - Reallabore der resilienzorientierten Transformation (Stadtentwicklung. Urban Development), S. 101-125

Potthast, T., 2015: Epistemisch-moralische Hybride!? Auf dem Weg einer Wissenschaftstheorie interdisziplinärer Ethik. In: Ammicht Quinn, R.; Potthast, T. (Hg.): Ethik in den Wissenschaften: 1 Konzept, 25 Jahre, 50 Perspektiven. Unter Mitarbeit von Birgit Kröber, Julia Dietrich, Jessica Heesen und Simon Meisch. Tübingen (Materialien zur Ethik in den Wissenschaften Bd. 10), S. 405-413

Schneidewind, U., 2014: Urbane Reallabore - ein Blick in die aktuelle Forschungswerkstatt. In: pnd online (III), S. 1-7; http://tinyurl.com/schneidewind-2014 (download 10.6.16)

WBGU - Wissenschaftlicher Beirat Globale Umweltveränderungen (Hg.), 2011: Welt im Wandel. Gesellschaftsvertrag für eine Große Transformation (Hauptgutachten). Berlin

\section{Kontakt}

Marius Albiez

E-Mail: marius.albiez@izew.uni-tuebingen

Andri König

E-Mail: andri.koenig@izew.uni-tuebingen.de

Thomas Potthast

E-Mail: potthast@uni-tuebingen.de

Internationales Zentrum für Ethik in den Wissenschaften (IZEW) an der Eberhard Karls Universität Tübingen

Wilhelmstraße 19, 72074 Tübingen

\section{Tentative Theses on Transformative Research in Real-World Laboratories}

\author{
First Insights from the Accompanying \\ Research ForReal ${ }^{1}$
}

by Niko Schäpke, Leuphana University of Lüneburg, Franziska Stelzer, Wuppertal Institute, Matthias Bergmann, ISOE Institute for Social-Ecological Research and Daniel J. Lang, Leuphana University of Lüneburg

Real-world laboratories are growing in popularity promising a contribution to both: the understanding and facilitation of societal transformation towards sustainability. Baden-Württemberg substantially funds real-world labs as part of the initiative "science for sustainability". To facilitate learning with and from these so-called BaWüLabs, they are supported by accompanying research conducted by two teams. This article presents first insights and theses on real-world labs as a research format, based in particular on the work of the accompanying research team ForReal. The team supports the labs in their realization and in providing general insights, e.g. by learning from related international research approaches and dialog with international experts, and analyzes suitable quality features and methods (the latter together with the University of Basel team). The theses presented here put up for discussion first insights on real-world labs as a transformative research approach and reflect on them from a theoretical perspective. They illustrate the relevance of a goal-oriented use of methods and present learning processes as core characteristics of real-world labs. The theses were formulated based on discussions with the BaWüLabs, exchange in international contexts as well as a thematic literature review.

Reallabore sind ein zunehmend populäres Forschungsformat, welches dazu beitragen soll, eine gesellschaftliche Transformation in Richtung Nachhaltigkeit sowohl zu verstehen als auch zu gestalten. Baden-Württemberg fördert 